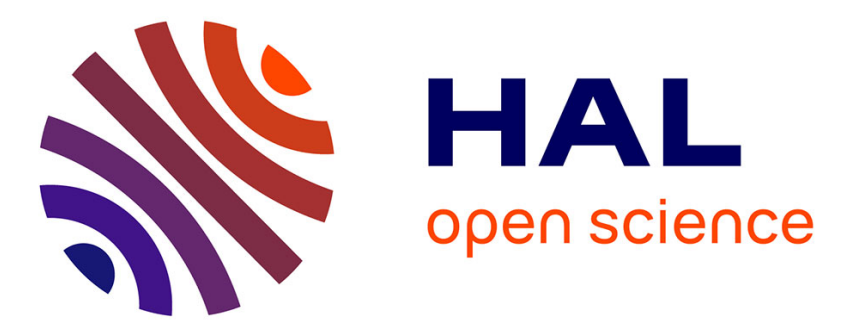

\title{
Multi-parametric 3D-point-spread function estimation in deep multiphoton microscopy with an original computational strategy dedicated to the reconstruction of muscle images
}

\author{
Claire Lefort, Emilie Chouzenoux, Laetitia Magnol, Henri Massias, \\ Jean-Christophe Pesquet
}

\section{To cite this version:}

Claire Lefort, Emilie Chouzenoux, Laetitia Magnol, Henri Massias, Jean-Christophe Pesquet. Multiparametric 3D-point-spread function estimation in deep multiphoton microscopy with an original computational strategy dedicated to the reconstruction of muscle images. SPIE Photonics Europe, Apr 2020, Online Only, United States. 10.1117/12.2554742 . hal-02566520

\author{
HAL Id: hal-02566520 \\ https://hal.science/hal-02566520
}

Submitted on 7 May 2020

HAL is a multi-disciplinary open access archive for the deposit and dissemination of scientific research documents, whether they are published or not. The documents may come from teaching and research institutions in France or abroad, or from public or private research centers.
L'archive ouverte pluridisciplinaire HAL, est destinée au dépôt et à la diffusion de documents scientifiques de niveau recherche, publiés ou non, émanant des établissements d'enseignement et de recherche français ou étrangers, des laboratoires publics ou privés. 


\title{
Multi-parametric 3D-point-spread function estimation in deep multiphoton microscopy with an original computational strategy dedicated to the reconstruction of muscle images
}

\author{
Claire Lefort*a, Emilie Chouzenoux ${ }^{\mathrm{b}}$, Laetitia Magnol ${ }^{\mathrm{c}}$, Henri Massias $^{\mathrm{a}}$, Jean-Christophe Pesquet $^{\mathrm{b}}$ \\ ${ }^{a}$ CNRS Institut de recherché XLIM, UMR 7258, Université de Limoges, Limoges, France \\ ${ }^{\mathrm{b}}$ Centre de la Vision Numérique, INRIA Saclay, CentraleSupélec, Université Paris-Saclay, France \\ ${ }^{\circ}$ GAAMA Lab, University of Limoges, France
}

*claire.lefort@xlim.fr

\begin{abstract}
Multiphoton microscopy (MPM) is an imaging method involving a near infrared range of excitation. However, generating 3D microscopic images in high depths with a satisfying quality is complex due to physical distortions of the beam (optical aberrations and diffraction limit) all long the optical path. The ability of computational approaches to restore reliably deep 3D images rests on the accuracy of the mathematical estimation of optical distortions introduced by the instrument. Instrumental distortions of the beam are characterized by the point spread function (PSF) evolution, especially in the optical axis and whose model can be fitted by a Gaussian shape. In this publication, we are presenting our homemade approach which rests first on the design of an optical phantom constituted by standardized microspheres, immobilized into a 3D volume of $2 \mathrm{~mm}$ deep. Then, 3D-volumes of images containing a single object are selected and isolated all along the sample depth using automatic morphological tools. Finally, our computational 3D-Gaussian shape fitting algorithm named FIGARO is applied on each individual PSF. FWHM evolution has thus bean measured for the first time in the 3 dimensions along a $2 \mathrm{~mm}$ depth. We have highlighted a highly significant effect of spherical aberrations in our MPM system, showing and increase with a factor 4 of the axial PSF dimensions in the depth while preserving the lateral PSF dimensions.
\end{abstract}

Keywords: Multiphoton microscopy, deep 3D-imaging, PSF evolution

\section{INTRODUCTION}

Three-dimensional (3D) imaging has been developed since 30 years for biomedical applications [1]. Imaging the whole organ in its physiological environment is of first interest for delivering correct diagnostics without any resection protocol and with a painless procedure. Magnetic resonance imaging (MRI) is the best known 3D and painless medical imaging method highly employed in hospitals. But the resolution of MRI is limited to the millimeter range and some details at the scale of the cell or at sub-cellular scale can be missed and can distort the end diagnostic. 3D, in vivo and in live imaging with a sub-micrometer resolution for biomedical applications is still a current necessity for biomedical researches and corresponds to a hot topic for biomedical imaging engineers [2, 3].

Optical microscopy is the softer solution for 3D biomedical imaging potentially in vivo and associated to a micrometer resolution. Today, the gold standard in optical microscopy is confocal microscopy where the samples imaged have to be labeled with fluorophores for revealing their presence [4]. This method is invasive due to the indispensable labeling process and is not the best solution for 3D and in depth imaging due to the use of UV or visible ranges of excitation. Such excitation range can be absorbed or scattered by the constituents of the sample imaged, which reduces the ability of such excitation wavelength to generate a deep image of biological targets. Moreover, UV or visible ranges are highly energetic, and can finally damage the sample imaged.

Multiphoton microscopy (MPM) appeared in 1990 as an alternative to confocal microscopy. MPM is a microscopy method based on the similar process of fluorescence emission to confocal microscopy. The specificity of MPM rests on the 
excitation strategy, based on the simultaneous interaction of two photons at least on the substance imaged. The 2 photons involved cumulate their energy for generating similar fluorescence emission to those involved in confocal microscopy. The two-photon approach opens the access to near infrared range (NIR) of excitation. Wavelengths in the NIR are less energetic, less absorbed and less scattered than UV and visible wavelengths. By consequence, NIR has a deeper penetration depth than UV and visible ranges and deep 3D and in vivo microscopy of biomedical samples become accessible [5]. Moreover, the simultaneous presence of two (or more) photons of excitation with the fluorophores is a phenomenon lowly likely. This characteristic results in an optical sectioning inherent to MPM [6].

MPM can reveals the structure of biomedical samples until several millimeters deep depending on the optical properties of the sample imaged, and especially its transparency to wavelengths in the NIR. However, optical microscopy and MPM are optical methods sensitive to physical phenomena distorting the excitation beam: diffraction limit and chromatic aberrations. These physical phenomena can be reduced thanks to computational strategies. Indeed, each microscope device has its own transfer function, also named Point Spread Function (PSF), whose consequences are harmful on the image quality [7]. Typically, optical distortions transform a Dirac signal into a Gaussian signal. The thinnest Gaussian shape is associated to the best resolution. But this property is an instrumental and mathematical challenge.

In this context, several computational strategies have been developed especially for the numerical compensation of optical distortions in MPM. Most of them rest on the idea that if the PSF of the system is known, its contribution to the image distortion can be reduced thanks to mathematical calculation such as deconvolution. Many plugin of Image $\mathrm{J}$ are proposing a strategy for a basic reconstruction of images based on the deconvolution of the measured image by the experimental PSF. However the 3D evolution is huge in the optical axis, as we are going to present in this publication. First, the interest of computational strategies in MPM is reminded. Then, we are quantifying the axial PSF evolution thanks to our computational strategy, presented hereafter. Finally, the illustration of the impact of axial PSF distortions is highlighted on images of muscle sample. We present an illustration of reconstruction of these images of muscle samples.

\section{INTEREST OF COMPUTATIONAL STRATEGIES IN MPM}

\subsection{Evaluation of the resolution}

MPM is an optical solution for the microscopic imaging of biological samples involving a minimally invasive procedure based on second and third orders light-matter interactions requiring NIR of excitation. However, the use of wavelengths in the NIR is associated to deleterious physical effects of light diffraction and chromatic effects whose impact on the image quality concerns essentially the resolution. In optical microscopy, the resolution is an essential parameter whose quantification is fixed by standard protocols. Considering that a Dirac signal is transformed into a Gaussian shape due to the specific transfer function of the optical microscope, the resolution estimation amounts to measure the wideness of the Gaussian signal. Experimentally, the Dirac signal is generated by fluorescent microspheres having a diameter smaller than the optical resolution. This Dirac signal goes through the optical microscope which applies its specific transfer function and acquisition parameters $\mathrm{H}$ and $\mathrm{w}$. By this procedure, the image of a point becomes a spread function; this transformation is thus named point spread function (PSF). Figure 1 illustrates the definition of the PSF and its impact on the images generated in optical microscopy. 


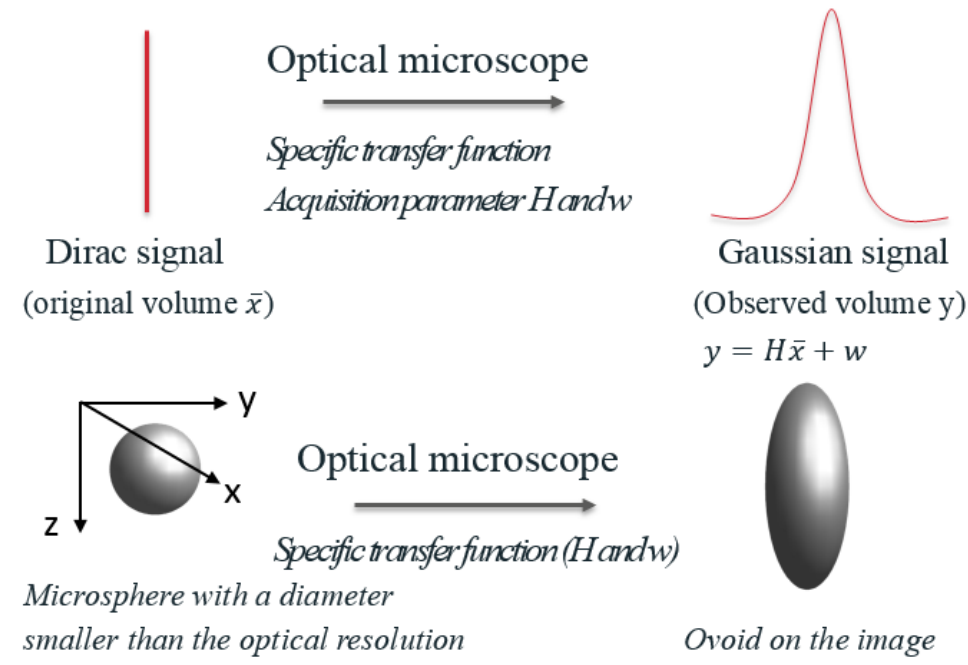

Figure 1. Numerical and experimental definitions of the PSF, the parameter governing resolution estimation in optical microscopy.

The true signal of the object, named $\bar{x}$ on Figure 1, passes through the instrument which induces a convolution by a function here named $\mathrm{H}$ associated with an acquisition noise $\mathrm{w}$. This convolution results in the observed volume $\mathrm{y}$. By consequence, the last signal is the convolution between the specific transfer function of the instrument and the original volume $\bar{x}$. Specific computational strategies can be developed in order to remove or reduce the instrumental contribution to the image. The main strategy consists in realizing a deconvolution calculation of the image by the PSF measurement. As the PSF measurement is not often possible exactly in the same experimental conditions as the image recording, a step of PSF fitting is often necessary and drives to the image reconstruction the closest to the true object $[8,9]$. That entails also to consider simultaneously the noise model.

\subsection{Quantification of resolution associated to a NIR of excitation in MPM}

Experimentally the resolution is a parameter that can be measured thanks to microspheres, standardized object with a shape known. Here, we are illustrating the resolution damages induced by the diffraction limit thanks to two kinds of colored microspheres: green and orange microspheres having a diameter of $0.2 \mu \mathrm{m}$. Table 1 presents the measured resolution thanks to a standard commercial solution of PSF estimation and image restoration: the commercial software Huygens, SVI. The two microspheres are imaged with our multiphoton microscope in standard excitation condition. 10 microspheres have been imaged for each measurement.

Table 1. Resolution measurement thanks to the PSF estimation and image restoration (Huygens, SVI).

\begin{tabular}{rcccc}
\hline & Raw PSF & \multicolumn{2}{c}{ PSF after deconvolution } \\
\hline Microsphere & $P S F(\mu \mathrm{m})$ & $P S F z(\mu \mathrm{m})$ & $P S F x y(\mu \mathrm{m})$ & $P S F z(\mu m)$ \\
Green & 0.58 & 1.82 & 0.31 & 0.78 \\
Orange & 0.61 & 2.13 & 0.34 & 1.35 \\
\hline
\end{tabular}

The diffraction limit has a nonhomogeneous impact on the image resolution depending on the considered plan. In the optical plan (xy), the resolution is not significantly damaged by physical phenomena. Nevertheless, the resolution in the 
optical axis (z) is hugely dependent to the diffraction limit: 17\% of increase for a raw PSF measurement between a green and an orange emission as shown in Table 1. By consequence, the NIR of excitation is necessarily associated to a damaged resolution compared, for example, to confocal or widefield fluorescence microscopy methods which involve a UV or visible excitation range.

\subsection{Quantification of resolution damages associated to deep imaging in MPM}

Even though MPM has a degraded resolution in the optical axis compared to standard confocal microscopy methods, the interest of MPM remains in its NIR of excitation. Indeed, biological samples contain constituents which are less absorbing and scattering NIR of excitation than UV or visible excitation. Thus, deeper 3D imaging windows are opened thanks to MPM. In that situation, we have quantified the resolution deterioration into a sample of 2-mm-depth. Thus, we have measured the PSF evolution into a sample of gel containing fluorescent microspheres with a diameter of $0.2 \mu \mathrm{m}$. The microspheres are spread homogenously into the $2 \mathrm{~mm}$ depth of the sample. Then, we have imaged the samples and cropped each PSF individually. Then, we have measured the PSF wideness at the full width at half maximum (FWHM). Figure 2 illustrates the evolution of the PSF dimensions in the optical plan (xy) and in the optical axis (z) in function with the depth of the PSF into the sample.

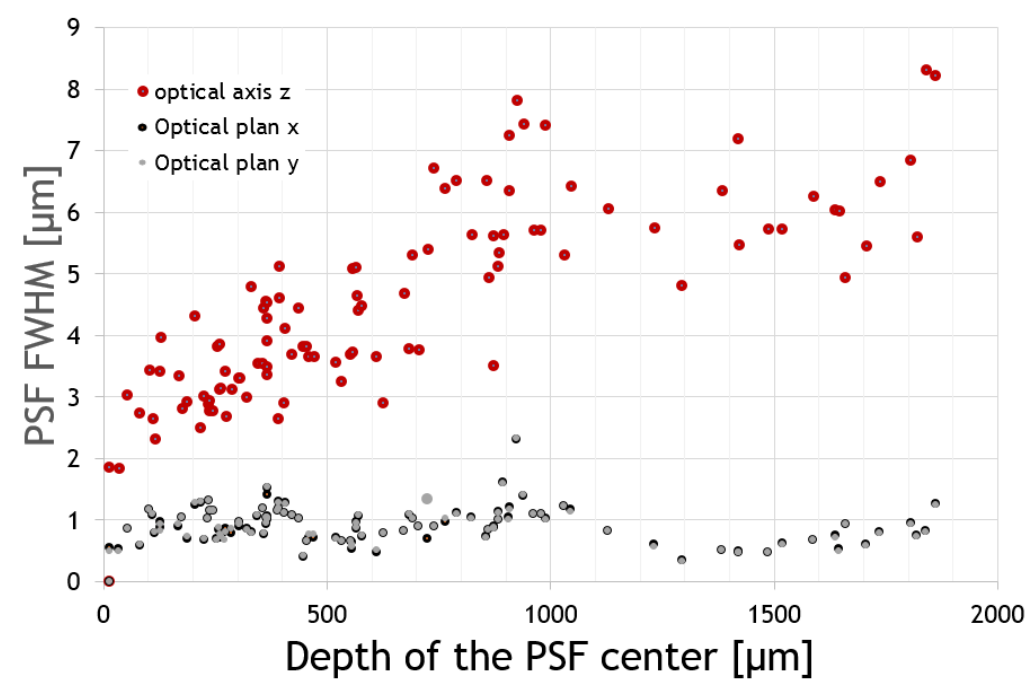

Figure 2. Measurement of the PSF FWHM in function with the depth of the PSF.

The evolution of the PSF dimensions in a 2-mm-deep sample is shown in Figure 2. On this graph, the PSF in the optical plan (xy) does not follow any significant evolution depending on their level of depth under the sample surface. However, the evolution of the PSF dimension, measured in the optical axis is hugely increased all along the 2-mm of depth. At the sample surface, the axial PSF is equal to $1.9 \mu \mathrm{m}$ at the FWHM. This value is in the expected standards. At $0.5 \mathrm{~mm}$ under the sample surface, the axial PSF has increased with a factor 2. At $2 \mathrm{~mm}$ under the sample surface, the wideness of the axial PSF has increased with a factor 4. This result, combined with the lateral PSF estimation, illustrates the consequent impact of spherical aberrations occurring in MPM. Spherical aberrations represent thus the physical phenomenon the more harmful for optical resolution in MPM. 


\subsection{Illustration of resolution damages associated to deep 3D imaging in MPM}

For illustrating the damages of resolution associated to deep 3D imaging of biomedical samples in MPM, the whole muscle is imaged with a standard solution of MPM. 2D images are recorded at many levels of depth with a fixed step of $0.1 \mu \mathrm{m}$. The stacks are then superimposed to generate the 3D image. Skeletal muscle is a good candidate for illustrating depth resolution damages, thanks to its striated structure, constituted by bundles of fibers, themselves constituted by a periodical assembly of myosin and actin merged alternatively and periodically. Different levels of periodicity can thus be imaged. Moreover, myosin is a protein organized in a noncentrosymmetric structure which generates a signal of second harmonic. The second harmonic generation (SHG) is emitted label-free by the structure and then collected by the detectors. Figure 3 presents the 3D image of the whole muscle. The image is generated with a field of view (FOV) of $1.6 \times 1.6 \times 0.7 \mathrm{~mm}^{3}$. Figure 3 left shows the 3D image with the standard $x y$ optical plan. Figure 3 right illustrates the 3D image in the $x z$ plan representing the optical axis.
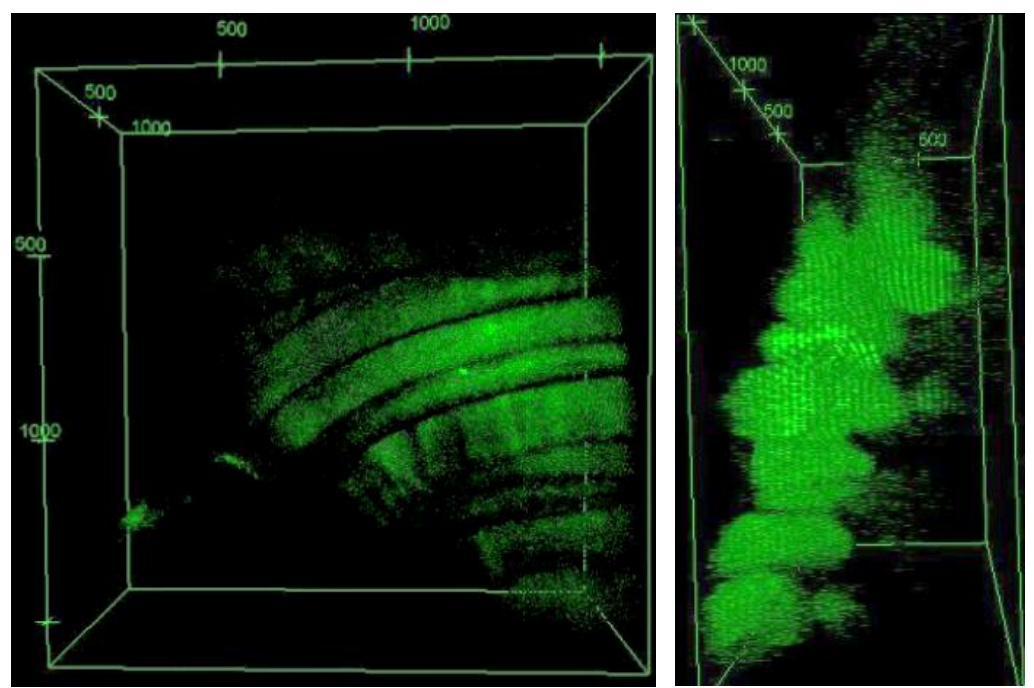

Figure 3. 3D images of a whole muscle. Label-free imaging of the myosin organization. Left image represents the front view of the image of the muscle sample, corresponding to the xy optical plan in the foreground. Right image represents the side view of the image of the muscle sample, corresponding to the xz plan illustrating the resolution deterioration in the optical axis.

Figure 3 right highlights several artefacts on the image. First, the 2D images which have been superimposed are revealed with vertical and luminous lines. Moreover, the emitted SHG is less precisely defined on the image in the depth and appears with luminous trails.

\subsection{Conclusion about the need in computational strategy in 3D MPM}

MPM is the exclusive solution offering 3D images of biological samples at millimetric depths and subcellular scale with a sub-micrometer resolution. But this optical method suffers from significant optical artefacts and especially spherical aberrations which damage the axial resolution of the 3D image, quantified by the PSF dimension in the optical axis (Figure 2). A realistic computational strategy for computational restoration of 3D MPM images generated along millimetric depths must consider the axial evolution of PSF. In that context, the quality of PSF estimation becomes the essential parameter for a successful image restoration in MPM. Noise and blur levels increasing with the depth are delivering to this topic a higher level of complexity when the PSF is located deeply under the sample surface. The space variant property of the PSF imposes to resort to an adapted computational strategies considering this variation in order to remove the PSF contribution to the image, especially in the optical axis. The solution that we have developed is based on a PSF fitting model which considers the spatial variation of the axial PSF in depth. 


\section{EXPERIMENTAL AND COMPUTATIONAL STRATEGY FOR PSF FITTING IN MPM WITH A 3D MULTI-PARAMETRIC SOLUTION}

\subsection{Experimental pipeline}

Our experimental strategy follows a protocol divided in five steps described thereafter. First, the whole muscle is resected from a wild mouse from tendon to tendon. Then, the muscle is fixed into a gel containing microspheres for PSF estimation. Individual images of microspheres are recorded and cropped. Simultaneously, the SHG signal from myosin of muscle is recorded. Each image of microsphere are then modeled with our FIGARO strategy (FItting GAussians with pRoximal Optimization). Finally, muscle images are deconvolved with our BP3MG solution (Block Parallel Majorize-Minimize Memory Gradient) considering the 3D evolution of the PSF depending on the depth of SHG recording.

\subsection{Computational strategy}

The computational estimation of the PSF Gaussian shape considers that each voxel $n \in\{1, \ldots N\}$ contained into the volume of interest must follow the shape $\mathrm{y}_{\mathrm{n}}$ :

$$
y_{n}=\bar{a}+\bar{b} \overline{p_{n}}+w_{n}
$$

w being the noise. Our strategy rests on the PSF fitting with a Gaussian shape whose expression is $\bar{p}_{n}$ :

$$
\bar{p}_{n} \approx \frac{|\bar{C}|^{\frac{1}{2}}}{(2 \pi)^{\frac{Q}{2}}} \exp \left(-\frac{1}{2}\left(x_{n}-\bar{\mu}\right)^{T} \bar{C}\left(x_{n}-\bar{\mu}\right)\right)
$$

The 3D variation of the PSF in the depth of the sample is considered by introducing a convolution operator $\mathrm{H}$ having a 3D variation in the depth and resulting in the measured signal y:

$$
y=H \bar{x}+w
$$

In this expression, $\bar{x}$ corresponds to the original volume. Figure 1 resumes the localization of these parameters all along the experimental pipeline.

Our computational approach proposes a new cost function for estimating $(\bar{a}, \bar{b}, \bar{p}, \bar{\mu}$, $\bar{C})$ from y data, based on a new alternating proximal algorithm with convergence guaranties. A penalized criterion $\mathrm{F}(\mathrm{x})$ is considered and includes the relative calculus bounds $\mathrm{R}(\mathrm{x})$ with the expression:

$$
F(x)=\frac{1}{2}\|H x-y\|^{2}+R(x)
$$

The mathematical details can be found in our previous publication [9]. Moreover, we have developed a version of this algorithm presented as a free plugin for Image $\mathrm{J}$. The plugin is available by following:

www.github.com/AlexisLauret/FIGARO.

\subsection{Reconstructed image of a muscle structure considering a space variant PSF}

The image size of the whole organ formed with the standard MPM protocol generates about 30 Go of raw data for a single image. No computational system can fairly well process simultaneously the whole image. By consequence, the illustration of our strategy is reduced at a box with dimensions of $22 \times 10 \times 10 \mu \mathrm{m}^{3}$. Figure 4 represents the resulting image which has been processed by our pipeline considering the axial variations of the PSF. 


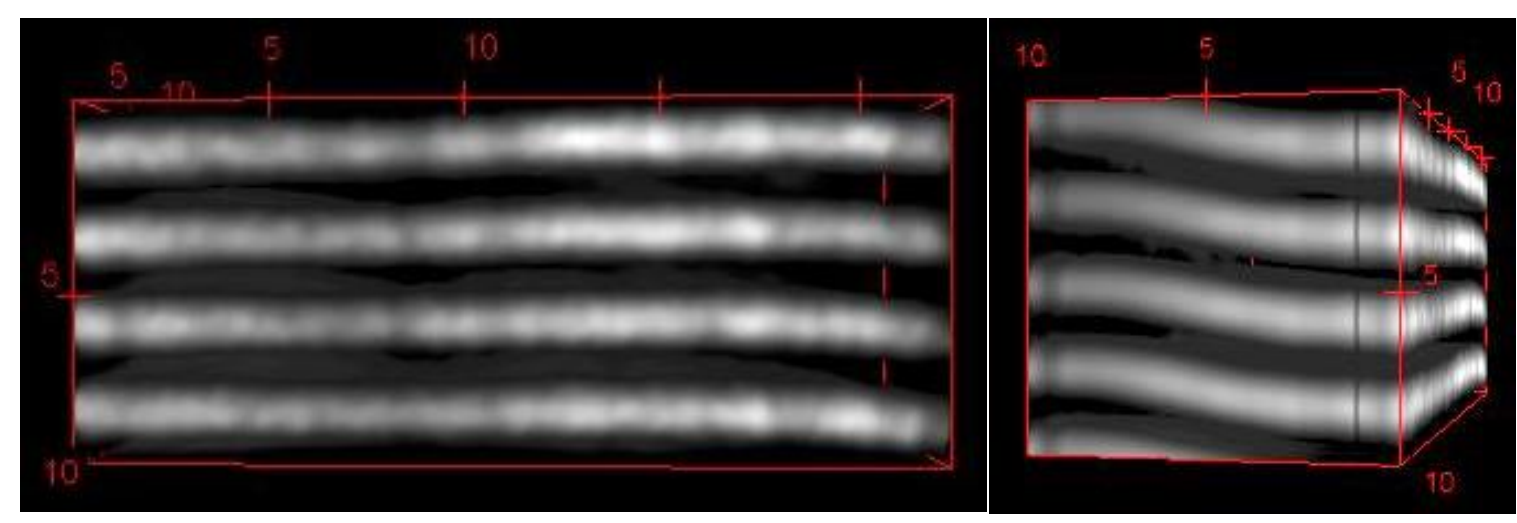

Figure 4. Muscle image of $22 \times 10 \times 10 \mu \mathrm{m}^{3}$ which has been processed by our experimental pipeline considering the axial variations of the PSF. Left image represents the xy optical plan in the foreground (front view). Right image represents the yz plan illustrating the interest of the image restoration in the optical axis (side view).

Figure 4 illustrates the interest of our computational strategy for image restoration especially in the optical axis, by considering the axial space variations of the PSF. Right image represents the side view of the muscle, contained into the yz plan. This view point illustrates the interest of the image restoration in the optical axis. Our computational strategy reveals the myosin organization all along the optical axis. Such a view point for muscle structure has never been shown in the experimental studies of muscle.

\section{CONCLUSION}

Multiphoton microscopy is an optical imaging method especially adapted to biomedical imaging. Its ability to produce 3D images is unequaled by the other imaging methods. Today, the main strategy chosen for generating 3D images of biomedical samples consists in resecting the sample of interest and associated this step to a labelling process with labeled antibodies. Such a time consuming, and costly solution, associated to image slicing and confocal image recording, generates only a partial description of the 3D structure of the muscle, missing out on details concerning the 3 dimensional organization. This property is especially true in muscle imaging where the $3 \mathrm{D}$ image reconstruction is often associated to a physical sample slicing. Here, we present our solution combining two technological advances: an instrumental one with a computational one. Instrumentally, we are benefiting to the non-centrosymmetric organization of myosin, a structural protein of muscle. This organization generates a second harmonic signal; myosin can thus be image label free in the whole depth of the muscle. Our computational strategy proposes a robust solution for point spread function fitting, which is usually deteriorated by noise, especially in the case of multiphoton excitation. Associated to an adapted deconvolution strategy, the robustness and reliable computational strategy shows a 3D organization of muscle which has never been demonstrated until now. 


\section{REFERENCES}

[1] J. Mertz, "Introduction to Optical Microscopy", Roberts \& Company Publishers (2009).

[2] S. F. Gibson, F. Lanni, "Experimental test of an analytical model of aberration in an oil-immersion objective lens used in three-dimensional light microscopy". J. Opt. Soc. Am. A, 8, 1601-1613 (1991)

[3] A. Diaspro, "Confocal and Two-photon microscopy: foundations, applications and advances", Wiley-Blackwell (2001).

[4] J. R. Lakowicz, "Principles of Fluorescence Spectroscopy", 3rd edn, Berlin: Springer (2006)

[5] B. R. Masters, P. T. So, "Handbook of Biomedical Nonlinear Optical Microscopy", Oxford University Press, ISBN 978-0-1951-6260-9 (2008)

[6] V. E. Centonze, J. G. White, "Multiphoton Excitation Provides Optical Sections from Deeper within Scattering Specimens than Confocal Imaging", 75 (4), 2015-2024 (1998)

[7] H. I. Labouta, M. Hampel, S. Thude, K. Reutlinger, K.-H. Kostka, M. Schneider, "Depth profiling of gold nanoparticles and characterization of point spread functions in reconstructed and human skin using multiphoton microscopy", J of Biomedical Optics, 5, 1, 85-96 (2012)

[8] X. Zhu, D. Zhang, "Efficient parallel Levenberg-Marquardt model fitting towards real-time automated parametric imaging microscopy", PLOS ONE, 8 (10), 1-9 (2013)

[9] E. Chouzenoux, T. T-K Lau, C. Lefort, J.-C. Pesquet, "Optimal Multivariate Gaussian Fitting with Applications to PSF Modeling in Two-Photon Microscopy Imaging", Journal of Mathematical Imaging and Vision 61 (7), 1037-1050 (2019) 